\title{
Dielectric-Based Temperature Sensing of Nanoliter Water Samples with a Post-Processing Tuned Matching Network
}

\author{
Gertjan Maenhout ${ }^{1}$, Tomislav Markovic ${ }^{2,1}$, Juncheng Bao ${ }^{1}$, \\ Georgios Stefanidis ${ }^{3}$, Ilja Ocket ${ }^{2,1}$ and Bart Nauwelaers ${ }^{1}$ \\ 1 Div. ESAT-Telemic, Kasteelpark Arenberg 10, Box 2444, 3001 Leuven, Belgium \\ 2 Imec, Kapeldreef 75, 3001 Heverlee, Belgium \\ 3 Chemical Engineering Department, KU Leuven, Celestijnenlaan 200F, Box 2424, \\ 3001 Leuven, Belgium
}

E-mail: gertjan.maenhout@esat.kuleuven.be

January 2020

\begin{abstract}
Microfluidic devices bring new opportunities in vast applications, in which the temperature control of nanoliter droplets is often required. Due to the inherit size of employed miniature volumes, several challenges exist related to temperature measurements. In this work, we present a non-contact and label-free dielectric-based temperature measurement technique. A capacitive sensor transduces the temperaturedependent dielectric properties into a capacitive value. With a post-processing tuned matching network, the reflection coefficient of the capacitive sensor is minimized at a specific frequency that is directly related to the sample temperature. The system was calibrated with a water-only calibration method using two calibration values. A measurement accuracy of $\pm 0.24^{\circ} \mathrm{C}$ was achieved with an offset of $-0.19^{\circ} \mathrm{C}$ in a temperature range from $20^{\circ} \mathrm{C}$ to $55^{\circ} \mathrm{C}$. The possibility to use the proposed sensor as a microwave heater at the same time, can lead to a dual-purpose device that minimizes the footprint of the chip and thus its cost, while also reducing the amount of electrical connections to the chip.
\end{abstract}

Keywords: microwave sensor; temperature measurement; temperature calibration; impedance matching; microfluidics

Submitted to: Meas. Sci. Technol. 


\section{Introduction}

Temperature control is indispensable in various microfluidic applications (Mark et al. 2010; Miralles et al. 2013). It has been widely used to improve reaction time and increase throughput and nowadays the influence of temperature control is being investigated to improve mixing (Yesiloz et al. 2017) as well as to optimize the generation of droplets (Yap et al. 2009). Both mentioned applications require an accurate temperature control system inside the microfluidic channel to ensure a reliable production. Additionally, these novel systems should rapidly heat the sample under test and have a low thermal time constant in order to boost the throughput.

Temperature control systems can be separated in a heating and a temperature sensing component. Several methods for temperature measurements exist in practice. In this introduction, we will briefly introduce some measurement techniques that are used in microfluidic applications. These techniques are generally divided into contact and noncontact based techniques. A first, contact based method using an electrical temperaturedependent resistor (RTD) (Tan et al. 2008; Zhong et al. 2009) is accurate but suffers from the intrinsic thermal time constant of the sensor. Furthermore, it can only measure the surface temperature that might be different from the bulk temperature of the liquid sample due to internal temperature gradients (Basu and Gianchandani 2007). Another contact based method used in microfluidic applications employs thermocouples (Gillot et al. 2006; Shibata et al. 2015), which again transduce the sample temperature into an equivalent electrical signal. They have all the disadvantages of RTD's, and additionally, their accuracy can be limited up to several degrees Celsius depending on the employed type (from $\pm 0.5^{\circ} \mathrm{C}$ for a type $\mathrm{T}$ thermocouple to $\pm 2.5^{\circ} \mathrm{C}$ for a type $\mathrm{J}$ thermocouple (Wu 2018)). Lately, thermistors have also been integrated in microfluidic applications (Bhargava et al. 2016). They have a similar accuracy as RTD's and are typically faster with a thermal response around $0.5 \mathrm{~s}$ when submerged in a stirred liquid. Nevertheless, they still measure surface temperature and their thermal time constant is still large. Finally, temperature measurements using fiber-optic measurement systems present a viable option that suffers less from a thermal time constant due to their miniature sensor size (Feng et al. 2011; Li et al. 2018). Nevertheless, they exhibit a worse accuracy in the range of several tenths of a degree Celsius.

A non-contact method using infrared temperature sensors (Keränen et al. 2010; Ryu et al. 2017; Yoo et al. 2008) poses no thermal loading to the system, which is unique to all up to now discussed techniques. Since this technique measures a physical property of the sample to determine its temperature, there is no transfer of thermal energy required to the sensor and therefore no thermal time constant is present. Although it has the advantage of not requiring physical contact, the accuracy also drops in the range of several degrees Celsius. In addition, temperature is also measured closer to the surface

rather than in the bulk as the infrared signal does not penetrate deep into liquid (Yoo et al. 2008). Another non-contact technique for temperature measurements employs temperature-dependent fluorescent dyes, such as Rhodamine B (Ross et al. 2001). Based 
Table 1: Overview of the characteristics of existing temperature sensing techniques found in literature.

\begin{tabular}{|l||c|c|c|c|c|}
\hline $\begin{array}{l}\text { Measurement } \\
\text { technique }\end{array}$ & $\begin{array}{c}\text { Sensor } \\
\text { placement }\end{array}$ & $\begin{array}{c}\text { Penetration } \\
\text { depth }\end{array}$ & $\begin{array}{c}\text { Temperature } \\
\text { accuracy }\left[{ }^{\circ} \mathrm{C}\right]\end{array}$ & $\begin{array}{c}\text { Thermal time } \\
\text { constant }\end{array}$ & $\begin{array}{c}\text { Calibration } \\
\text { method }\end{array}$ \\
\hline RTD & direct contact & surface & \pm 0.1 & up to $100 \mathrm{~s}$ & 3 cal. meas. \\
\hline Thermocouple & direct contact & surface & $\pm 0.5-2.5$ & $1.5 \mathrm{~ms}$ & physical property \\
\hline Thermistor & direct contact & surface & \pm 0.1 & $0.5 \mathrm{~s}$ & 3 cal. meas. \\
\hline Fiber-optic & direct contact & surface & \pm 0.3 & $108 \mathrm{~ms}$ & 2 cal. meas. \\
\hline Infrared & contactless & $16 \mu \mathrm{m}$ & \pm 0.5 & $\mathrm{~N} / \mathrm{A}$ & 3 cal. meas. \\
\hline Fluorescent dye & dye in sample & volumetric & $\pm 0.03-3.5$ & $33 \mathrm{~ms}$ & 1 cal. meas. + lit. \\
\hline
\end{tabular}

on the initial reflected measured intensity at a measured start temperature, the change in temperature can be tracked by following the change in reflected intensity as stated in literature (Ross et al. 2001). This method allows spatial temperature measurements with accuracy of several degrees Celsius at the cost of optical access to the investigated liquid. Notwithstanding that it offers the benefits of spatial temperature measurements, it is not always possible to dilute a fluorescent dye in a liquid sample as it can hinder optical readouts of biological processes and interfere with biomedical experiments, e.g. cell culture growth (Kaji et al. 1991). Table 1 contains a summary of the presented techniques with an overview of some of their characteristics. This summary demonstrates that there exists an opportunity for a novel technique which is volumetric and fast while achieving a temperature accuracy in the range of several tenths of a degree Celsius.

With respect to the heating part of temperature control systems, dielectric microwave heating is used more and more for rapid, volumetric and contactless heating of samples (Markovic et al. 2016). This microwave heating technique introduces an extra problem for direct contact temperature measurement techniques: contact-based sensors influence the dielectric distribution within the heated volume and thus also impact the final temperature distribution (Estel et al. 2017). This influence on the dielectric distribution again results in an extra disadvantage of direct contact methods in microwave heating applications. During the investigation of microwave heating of water, the complex permittivity of liquids, e.g. water and water mixtures (Ellison 2007; Peyman et al. 2007; Sato and Buchner 2003), was also characterized in more detail and it is clear that its dielectric behavior strongly depends on temperature. This behavior complicated the design of microwave heaters over a broad temperature range since the load impedance of the heating system becomes temperature-dependent. Nevertheless, it does allow permittivity-based temperature measurement of the sample-under-test. The possibility of using the heater itself as a temperature sensor, allows constant temperature monitoring of the sample during heating and it reduces the footprint of the chip. Additionally, dielectric-based temperature sensing is a non-contact method due to the penetration of the electromagnetic waves, its speed is comparable to the one of optical temperature sensors and it does not require any labeling.

The concept that temperature changes influence the complex permittivity of a 
substrate and thus causes a shift in the resonant frequency of embedded resonators has been used to construct wireless temperature sensors for high-temperature applications (Cheng et al. 2012; Lu et al. 2018). Dielectric-based temperature sensing has been further developped for temperature measurements of single phase water droplets in microfluidic applications (Wong et al. 2016). Here, the multiple advantages of dielectric temperature sensing were fully exploited for the first time. However, the used split ring resonator sensor in (Wong et al. 2016) is relatively large compared to the microfluidic chip. This large area leaves no more space to add more functionality on chip, e.g. microwave biosensing (Bao et al. 2019; Grenier et al. 2009), which increases overall cost. Since one of the bottlenecks for the upscaling of microfluidic devices is directly related to the economies of scale (Mohammed et al. 2015), it makes sense to migrate towards the use of inexpensive and disposable microfluidic chips connected to reusable components on a permanent, off-chip setup (Bao et al. 2020; Maenhout et al. 2019a). Recently, we demonstrated that the combination of a miniature sensor on-chip with computational power off-chip enables the dielectric temperature measurement of a water droplet (Maenhout et al. 2019b). Moreover, if the functionality of a microwave heater and a dielectric thermometer are combined in a single miniature dual-purpose device, the footprint of the chip will decrease and the number of connections and interconnects will be reduced. Such dual-purpose devices will be cost-beneficial and close the gap towards inexpensive and disposable chips on a reusable setup for biomedical applications (Esfandyarpour et al. 2017).

In this work, we propose a novel dielectric-based temperature sensing method for nanoliter water samples in microfluidic applications. The temperature extraction is based on our previously developed water-only calibration technique. The method uses an external, reusable hardware board with an embedded matching network to transform the impedance of the sensor into a matched load. The temperature of the sample is related to a specific frequency where the load is matched. Section 2 elaborates more on the used materials and methods. The obtained results are presented in section 3 and are further discussed in section 4. At last, the main achievements will be summarized in the conclusion in section 5 .

\section{Materials and methods}

\subsection{Selection of the operating frequency}

In dielectric-based temperature sensor design, all parameters are determined by the complex permittivity value: $\epsilon=\epsilon^{\prime}-j \epsilon^{\prime \prime}$. The dominant parameter for frequency selection is $\epsilon^{\prime}$, since it directly influences the capacitance value of the sensor loaded with the sample. These values are presented in Figure 1a for deionized water, based on the equation provided by Ellison (Ellison 2007). To obtain the most sensitive measurements, the difference $\Delta \epsilon^{\prime}$ per ${ }^{\circ} \mathrm{C}$ should be maximized at the operating frequency. It can be observed that up to $2.5 \mathrm{GHz}, \epsilon^{\prime}$ changes significantly under influence of the temperature. 


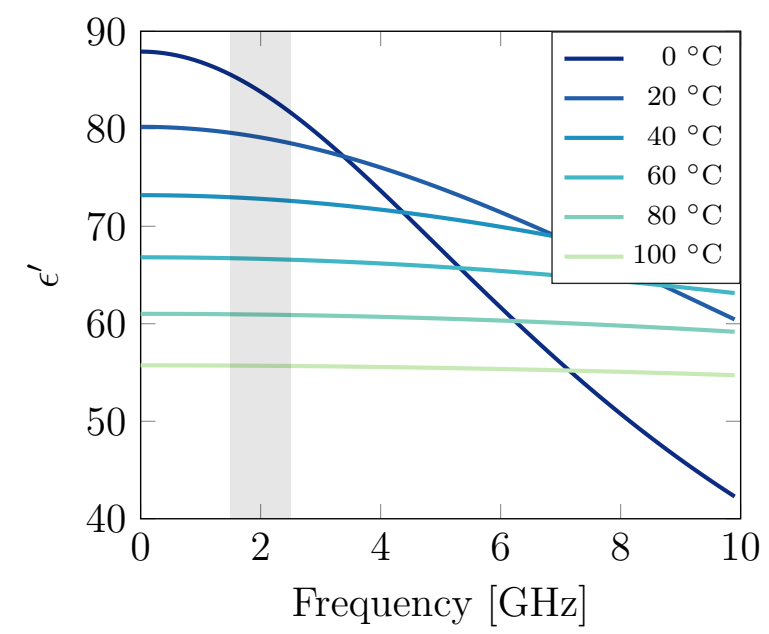

(a)

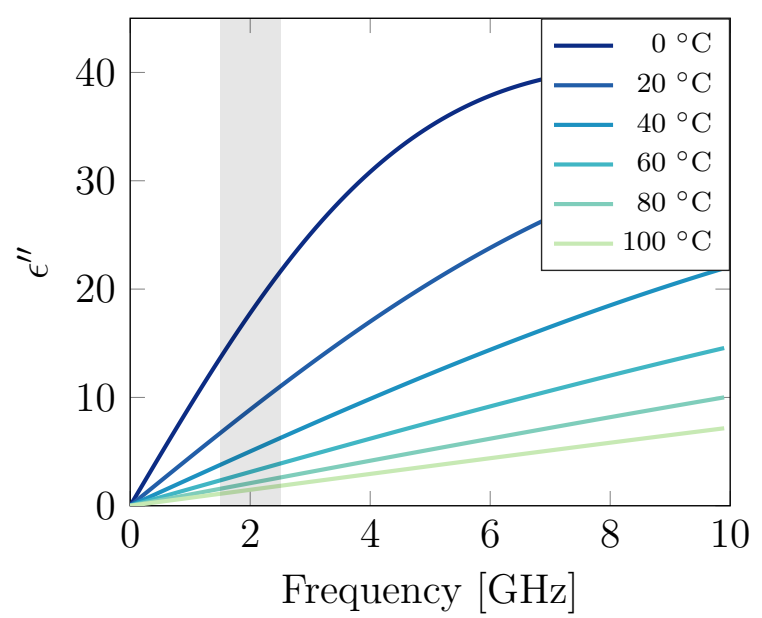

(b)

Figure 1: The complex permittivity $\epsilon=\epsilon^{\prime}-j \epsilon^{\prime \prime}$, with the real $\epsilon^{\prime}$ and imaginary $\epsilon^{\prime \prime}$ part in respectively (a) and (b), of deionized water for frequencies ranging from DC to $10 \mathrm{GHz}$ and for multiple temperatures. The shaded area indicates the behavior of the complex permittivity in the frequency range of interest. (based on the work from Ellison (Ellison 2007))

Afterwards, the difference $\Delta \epsilon^{\prime}$ per ${ }^{\circ} \mathrm{C}$ diminishes and the $\epsilon^{\prime}$ curves start to overlap, which creates an ambiguity in the measurement system that could result in a faulty temperature read-out.

A second parameter to consider for frequency selection is the imaginary part of the complex permittivity $\epsilon^{\prime \prime}$, shown in Figure 1b. This parameter governs the dielectric losses that are responsible for the heating of the sample. In this research domain, $\epsilon^{\prime \prime}$ should be as low as possible since heating the liquid sample should be avoided during temperature measurements. This criterion indicates that the lowest possible frequency is the most favorable one in the case of pure water.

Lastly, the size of the complete setup is the final parameter to take into consideration during frequency selection as a higher operating frequency will shrink the size of the wavelength-related structures. This higher operating frequency automatically results in a smaller footprint and thus reduces the overall system cost. The optimal operating frequency can be selected with a weighted cost function involving sensitivity as well as manufacturing and instrumentation costs. While the presented technique is valid over a much broader frequency range, we presented in this work a proof of concept of this novel temperature sensing technique while pursuing a small sensing setup. Therefore, an operating frequency around $2 \mathrm{GHz}$ is chosen as a compromise between the change of the real part $\Delta \epsilon^{\prime}$ per ${ }^{\circ} \mathrm{C}$, the relatively low imaginary part $\epsilon^{\prime \prime}$ and the wavelength at the operating frequency. 


\subsection{Interdigital capacitive sensor}

A capacitive sensor transduces the dielectric properties of the sample in a measured capacitive value. In this work, we opted for an interdigital capacitor (IDC) topology in coplanar waveguide (CPW) technology to implement the sensor due to three reasons. First, an IDC has a volumetric field distribution that penetrates the droplet in a nonintrusive way. Secondly, the IDC in CPW technology is a structure in a single plane that can be fabricated with an inexpensive process and it allows smooth integration with the nowadays existing microfluidic platforms. At last, the designed IDC is a one-port structure, which simplifies the setup and reduces the number of required connections to the measurement equipment.

The most important design value of the IDC is its capacitance value. A larger capacitance value of the IDC results in a higher sensitivity of the temperature measurement system. A straightforward procedure to increase the capacitance is increasing the length $L$ of the IDC fingers. However, if $L$ becomes comparable to the wavelength, the electromagnetic field along the fingers becomes less uniform, as we have observed during the design in COMSOL Multiphysics simulations. This non-uniform field distribution results in a less homogeneous measurement zone that prioritizes the temperature of specific zones, which is not desired. Therefore, $L$ is chosen an order smaller than the wavelength at $2 \mathrm{GHz}$ for a $20^{\circ} \mathrm{C}$ water $\left(\epsilon^{\prime} \approx 80\right)$ loaded IDC on a quartz $\left(\epsilon^{\prime} \approx 4\right)$ wafer and $L$ is thus limited to $400 \mu \mathrm{m}$. The finger width $W$ and gap $G$ are chosen at $30 \mu \mathrm{m}$, which allows the electromagnetic fields to penetrate the liquid theoretically up to $60 \mu \mathrm{m}$ (Mamishev et al. 2004). A sensing depth of $60 \mu \mathrm{m}$ is adequate since it corresponds with the height of channels used in microfluidic platforms. If microfluidic channels with a greater height are used, the sensing volume can be adapted by tuning the values $W$ and $G$ of the IDC (Mamishev et al. 2004). The sensing volume of this manufactured chip equals $L \times 13 W \times 14 G=19.44 \times 10^{-12} \mathrm{~m}^{3}=19.44 \mathrm{nl}$. In the simulations, the IDC is covered with a water droplet that is larger than the

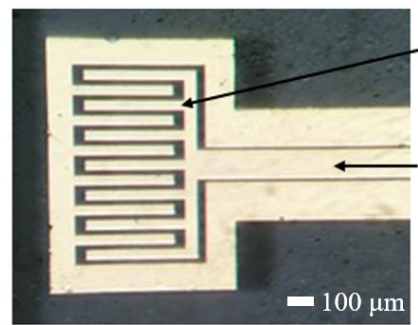

(a)
Electrode width and spacing of $30 \mu \mathrm{m}$

Line - gap - ground width of CPW feed $110-10-170 \mu \mathrm{m}$

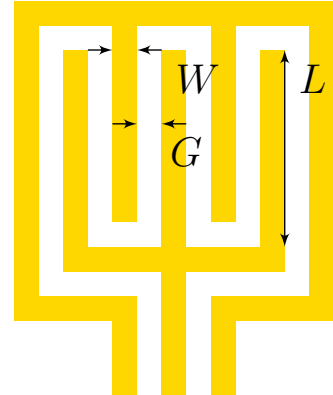

(b)

Figure 2: (a) Photograph of the manufactured gold-on-quartz interdigital capacitor and its feeding line in coplanar waveguide with corresponding dimensions. (b) Schematic overview of the interdigital capacitor indicating its length $L$, the finger width $W$ and the gap between the fingers $G$. 
sensing volume such that the shape and the size of the droplet have no impact on the simulation results. In the conducted experiments, a larger sample volume $(30 \mu \mathrm{l})$ is used to eliminate the effects of shape and size as well. A 13-finger IDC loaded with such a $20^{\circ} \mathrm{C}$ water droplet results in a capacitance value of $5 \mathrm{pF}$, which proved to be sufficient to achieve the targeted accuracy of $1^{\circ} \mathrm{C}$ in Keysight Advanced Design System (ADS) Momentum simulations.

The IDC is fabricated in the cleanroom of Leuven-NanoCentre. A quartz substrate is chosen due to its low $\tan \delta$ at microwave frequencies, which results in low dielectric losses in the substrate itself. A photoresist is spin-coated on the wafer and patterned using a UV-lithography step. Next, a $50 \mathrm{~nm}$ chromium layer is deposited on the wafer. This chromium layer is used to promote better adhesion between gold and quartz. A $400 \mathrm{~nm}$ gold layer is deposited afterwards. At last, the resist is removed resulting in the gold IDC pattern on the quartz wafer. The manufactured IDC is shown in Figure 2 along with the corresponding dimensions. After the manufacturing of the chip, a polydimethylsiloxane (PDMS) ring is bonded to the quartz substrate to guarantee a stationary and repeatable position of the liquid sample throughout multiple measurements. At last, a subminiature version A (SMA) end-launch connector is soldered to the chip to enable a standard interface with measurement equipment.

\subsection{Operating principle}

The technique uses a capacitive sensor, the aformentioned IDC, to transduce the temperature-dependent dielectric properties of the sample into a capacitive value. The technique works in the same frequency band as our previous work (Maenhout et al. 2019b) but an extra hardware board with a matching network (MN) is added. This additional board relaxes the software requirements specific to the previously investigated two-tier temperature extraction technique since this technique only requires to find a

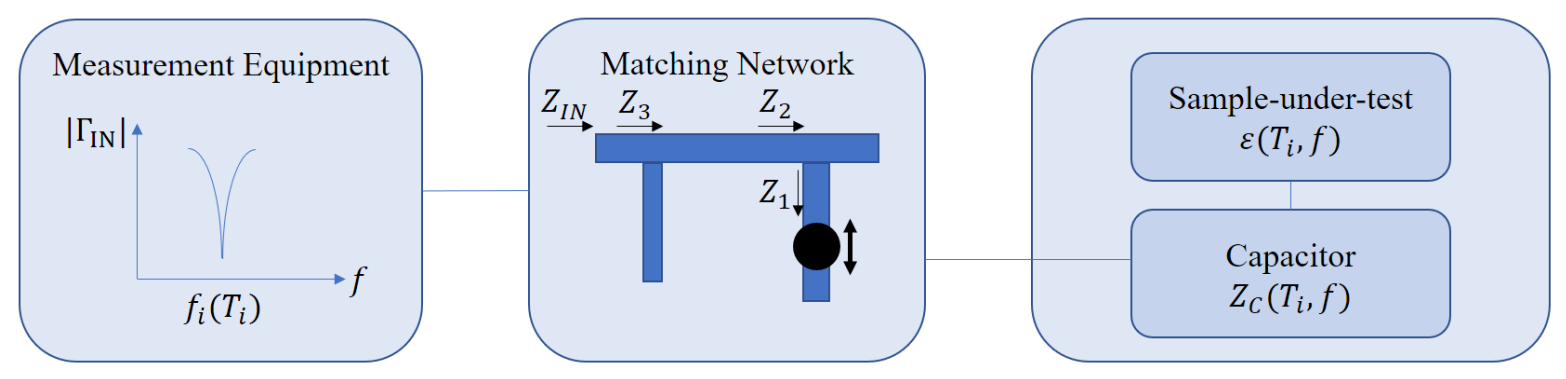

Figure 3: The block schematic of the proposed measurement technique: the temperature of the sample-under-test $T_{i}$ determines the permittivity of the sample. The capacitor transduces the temperature dependent permittivity into a capacitance value. The MN transforms the impedance $Z_{C}$ to impedance $Z_{I} N$ following equations (1)-(5) where the electrical length $\beta \times L_{a}$ can be altered by adjusting the position of the pad (black dot). The measurement equipment tracks $\left|\Gamma_{I N}\right|$ over the frequency domain and determines the temperature-dependent, matched frequency $f_{i}\left(T_{i}\right)$ where $\left|\Gamma_{I N}\right|$ is minimal. 
minimum value whereas our previous technique relies on solving a least square curve fitting algorithm. A complete overview of the system used in this technique is shown in Figure 3. Here, a clear distinction is made between the sensing part on a disposable chip versus the reusable part off-chip: a sensing capacitor is placed on chip and aligned with the fluidics to properly position the sample-under-test, while the MN is placed off-chip. The impedance of the capacitive sensor $Z_{C}$ is determined by the temperature-dependent permittivity of the sample $\epsilon(T, f)$ :

$$
Z_{C}(T, f)=\frac{-j}{2 \pi f \times C(\epsilon(T, f))} .
$$

$Z_{C}$ is transformed by a double stub MN. The first open-ended stub (electrical length $\beta \times L_{a}$, line impedance $Z_{a}$ ) transforms an open into impedance $Z_{1}$ :

$$
Z_{1}=\frac{Z_{a}}{j \times \tan \left(\beta \times L_{a}\right)},
$$

which is placed in parallel with $Z_{C}(T, f)$ :

$$
Z_{2}(T, f)=\frac{Z_{C}(T, f) \times Z_{1}}{Z_{C}(T, f)+Z_{1}} .
$$

Next, a transmission line (electrical length $\beta \times L_{b}$, line impedance $Z_{b}$ ) between both stubs transforms this aforementioned parallel impedance to impedance $Z_{3}$ :

$$
Z_{3}(T, f)=Z_{b} \times \frac{Z_{2}+j \times Z_{b} \tan \left(\beta \times L_{b}\right)}{Z_{b}+j \times Z_{2} \tan \left(\beta \times L_{b}\right)} .
$$

Lastly, the second open-ended stub (electrical length $\beta \times L_{c}$, line impedance $Z_{c}$ ) is once again added in parallel with impedance $Z_{3}$ which results in $Z_{I N}(T, f)$ :

$$
Z_{I N}(T, f)=\frac{\frac{Z_{c}}{j \times \tan \left(\beta \times L_{c}\right)} \times Z_{3}}{\frac{Z_{c}}{j \times \tan \left(\beta \times L_{c}\right)}+Z_{3}} .
$$

The measurement equipment measures the input reflection coefficient of the input impedance $Z_{I N}(f, T)$ that depends on the frequency $f$ and the temperature $T$, with respect to $50 \Omega$ :

$$
\Gamma_{I N}(T, f)=\frac{Z_{I N}(T, f)-50 \Omega}{Z_{I N}(T, f)+50 \Omega} .
$$

This input impedance is measured by the measurement equipment, in this work a vector network analyzer (VNA), as an input reflection coefficient $\Gamma_{I N}$. The measured magnitude of the input reflection coefficient $\left|\Gamma_{I N}(T, f)\right|$ will have a minimum at a frequency point $f_{i}$ related to the temperature $T_{i}$ of the liquid sample and $f_{i}$ changes accordingly to changes in $T_{i}$.

\subsection{Post-processing tuned matching network}

A matching network is added to the measurement system to bring the impedance of the capacitive sensor to $50 \Omega$. We did not opt to use lumped components due to the large component uncertainty. Therefore, passive microwave transmission lines and parallel 
stubs are chosen for the MN topology because their behavior is well-characterized in microwave design over a broadband frequency range and they come at an affordable system cost. As explained in multiple microwave engineering courses, every load can be transformed to $50 \Omega$ with a transmission line and a single stub (Pozar 2012). However, because the MN is connected off-chip, space restrictions are less important. The larger the MN becomes, the more narrow-banded it will behave and if the magnitude of the reflection coefficient becomes more narrow-banded, the matched frequency can be obtained with an increased accuracy. Therefore, a second parallel stub in the MN topology design is added since the area is available and since simulations demonstrated that this enlarged the shift in matched frequency per ${ }^{\circ} \mathrm{C}$.

To include the effects of the PDMS ring, the feeding transmission line and the SMA end-launch coaxial connector in the MN design, the MN layout is designed based on the measured impedances of the water loaded IDC sensor at different temperatures. Once these measured impedances are obtained, the $\mathrm{MN}$ is designed in ADS Momentum. To further close the gap between simulations and the manufactured board, the substrate parameters (Rogers 4350B laminate) and a model of the microwave connector (Amphenol RF 132432 SMA end-launch connector) at the interface of the MN, were obtained using the reported calibration structures and methods (Mandic et al. 2012; Reynoso-Hernández et al. 1999).

However, due to the manual assembly process of the connectors and possible overetching of stubs and transmission lines during the manufacturing process, discrepancies between simulations and measurements often occur and cause a deviation of the measured input impedance with respect to the desired $50 \Omega$. Therefore an additional post-processing tuning step of the $\mathrm{MN}$ is performed after manufacturing to counter the effect of these deviations. A small metal pad with a radius of $3 \mathrm{~mm}$ is slid over the open-ended stub is used for post-processing tuning of the MN. Adjusting the position of the pad with respect to the open-ended part of the stub, $L_{1}$, is equivalent to changing the physical length of a regular open-ended stub. This concept is graphically illustrated in Figure 4. On the right hand side of the figure, an equivalent model for the open-ended stub with the metal pad at position $L_{1}$ is presented. Since the dimensions of the metal pad are small compared to the wavelength at $2 \mathrm{GHz}$, we can consider it as a lumped component, i.e. a shunt capacitor $C$. The two transmission lines and the capacitor will together contribute to the phase of the aforementioned stub impedance $Z_{1}$. Although the contribution of the two transmission lines is fixed due to their length, the contribution of the capacitor $C$ to the phase of the stub impedance $Z_{1}$ will depend on its placement. The closer the pad is placed near the end of the stub, the larger the contribution of $C$ will be to the phase of $Z_{1}$ (similar to $L_{1}^{\prime}$ in Figure 4). The further the pad is placed, the smaller the contribution of $C$ to the phase of $Z_{1}$ (similar to $L_{1}^{\prime \prime}$ in Figure 4), as can be deduced from equation 7 :

$$
Z_{y}=-j \frac{Z_{a}}{\tan \left(\beta L_{1}\right)+\left(2 \pi f \times C \times Z_{a}\right)}
$$

This $L_{1}$-dependent change in phase of $Z_{1}$ is equivalent to a rotation around the 


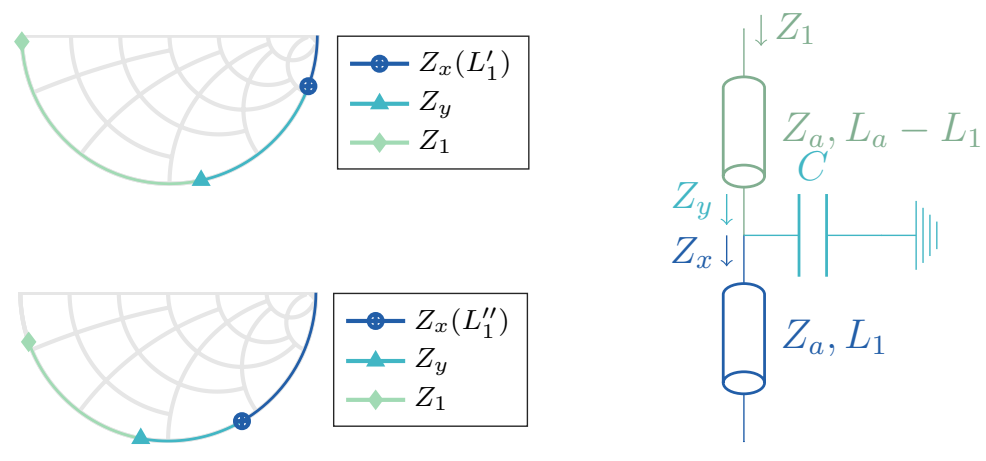

Figure 4: The equivalent circuit model of the stub with the metal pad is shown on the right. Tuning the length $L_{1}$, while keeping the total length $L_{a}$ constant, alters the effect of the fixed shunt capacitor $C$ as shown on the Smith charts for $L_{1}^{\prime}$ and $L_{1}^{\prime \prime}$ with a fixed total length $L_{a}$ and shunt capacitance $C$.

Smith chart which corresponds to changing the length of a regular open-ended stub.

$$
Z_{1}=Z_{a} \frac{Z_{y}+j Z_{a} \times \tan \left(\beta\left(L_{a}-L_{1}\right)\right)}{Z_{a}+j Z_{y} \times \tan \left(\beta\left(L_{a}-L_{1}\right)\right)}
$$

In Figure 5, the matching network is simulated in ADS Momentum with the pad at different positions of $L_{1}$. On both sides, the previously deduced connector model is virtually assembled and the connectorized $\mathrm{MN}$ is terminated at the right hand side with the measured impedance of the IDC, loaded with a water sample at a temperature of $22.5^{\circ} \mathrm{C}$. The pad is virtually slid over the stub from $L_{1}$ equal to $0 \mathrm{~mm}$ to $5 \mathrm{~mm}$. This simulation demonstrates that this post-processing step can bring the input impedance

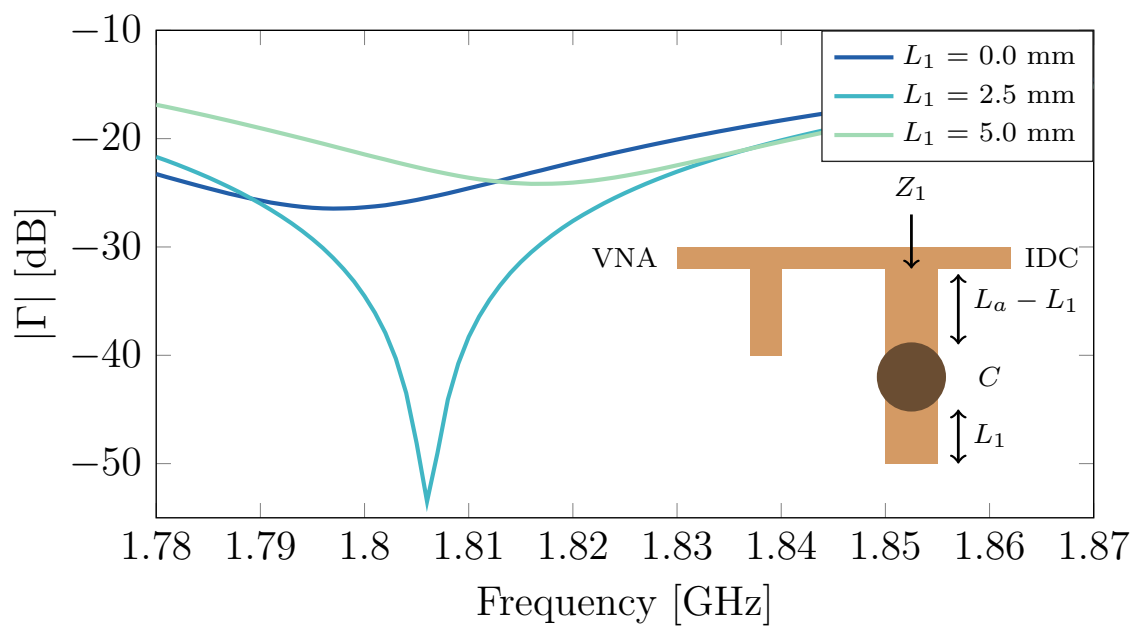

Figure 5: Simulations of the reflection coefficient of the MN with the pad at different positions $L_{1}$, connectorized with the derived connector models and terminated with the measurement of the impedance of the IDC when loaded with a sample at $22.5^{\circ} \mathrm{C}$. On the bottom right side, a sketch of the tunable $\mathrm{MN}$ is presented. 
closer to $50 \Omega$ at a temperature-specific frequency and thus can successfully compensate for manufacturing artifacts.

\subsection{Measurement setup}

The quartz chip with the IDC is placed on an aluminum block with an embedded, regulated resistive heater and it is heated to multiple temperatures. At each temperature step, the temperature of the sample in the PDMS ring is monitored directly with a type $\mathrm{K}$ thermocouple that has a relative accuracy of $\pm 1{ }^{\circ} \mathrm{C}$. The thermocouple is placed in the liquid sample under test in the PDMS ring, $2 \mathrm{~mm}$ away from the IDC itself to ensure that it is not being sensed by the IDC and thus does not influence the permittivity-based temperature measurements. The complete setup is put in a woodenpolymethylmethacrylate (PMMA) enclosure. The enclosure is sealed to limit the air circulation from the laboratory temperature airflow system to the liquid sample. This sealing reduces the speed of evaporation of the liquid sample. A small opening, which

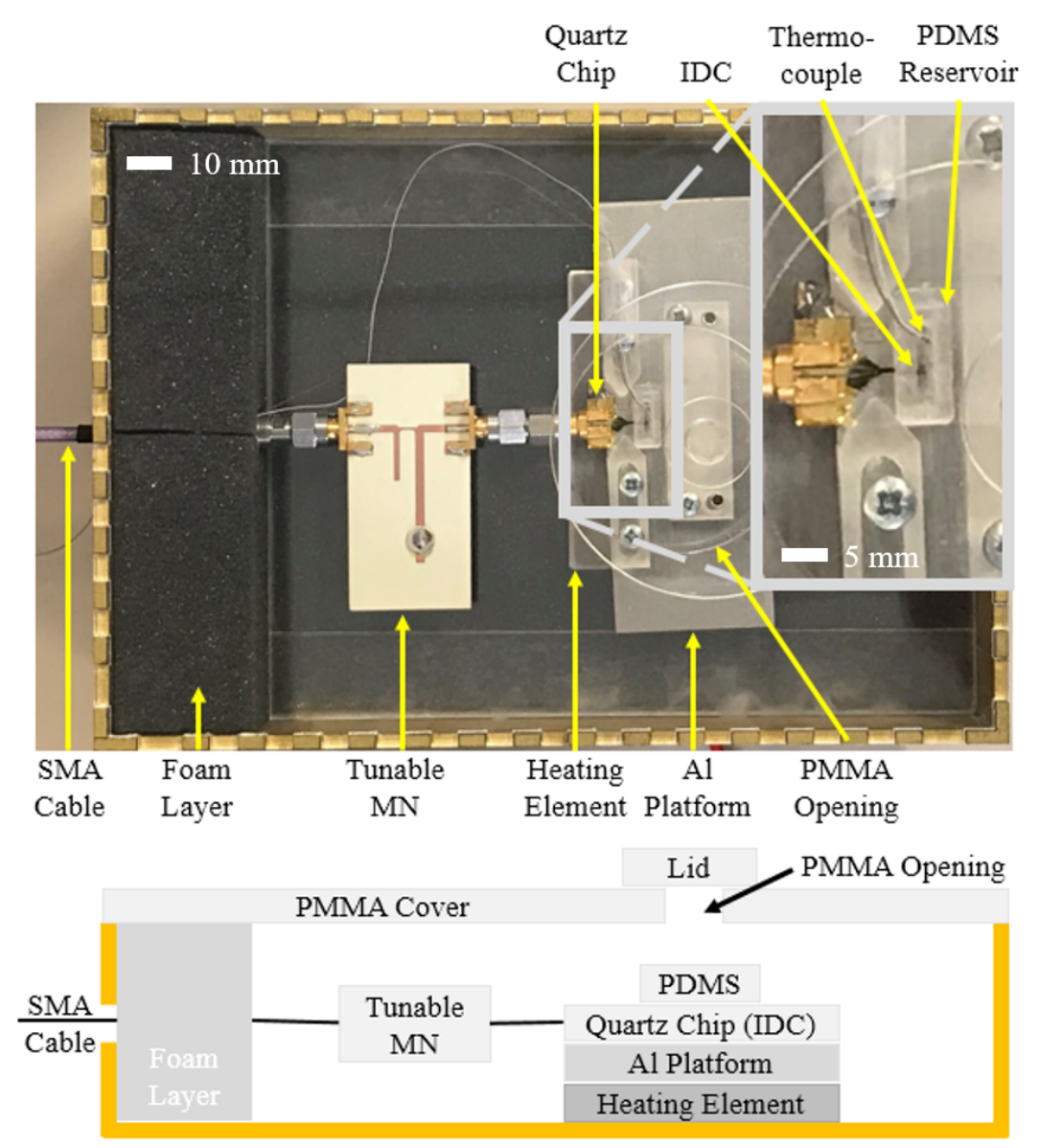

Figure 6: The measurement setup in the wooden-PMMA enclosure with a magnification of the IDC, the PDMS reservoir and the thermocouple positioned in the PDMS reservoir (TOP). A schematical side view of the setup is presented (BOTTOM). 
can be closed with a lid, is present in the PMMA cover and is used for adding extra liquid in the PDMS reservoir to compensate for evaporated liquid. The SMA cable and the thermocouple are brought into the enclosure through a foam layer. This layer adapts its shape to the cables and thus limits the exposure to air flow.

All measurements were conducted with a Keysight PNA (E8361A). A system calibration with a standard open-short-load calibration kit placed the reference plane at the SMA-connecter of the matching network. The intermediate frequency bandwidth was set to $100 \mathrm{~Hz}$ and a low output power level of $-10 \mathrm{dBm}$ was applied to avoid effects of dielectric heating (Bao et al. 2017). The PNA frequency range was set from $1.78 \mathrm{GHz}$ to $1.87 \mathrm{GHz}$ with a frequency step of $0.1 \mathrm{MHz}$.

\section{Results}

After the aforementioned calibration of the PNA, the reflection coefficient was measured during three separate experiments. In each experiment, the reflection coefficient is measured at eight different temperatures in the range from $20^{\circ} \mathrm{C}$ to $55^{\circ} \mathrm{C}$. The measured reflection coefficients for the eight temperatures of the first experiment are presented in Figure 7. Because the aforementioned post-processing of the MN took place at room temperature, the lowest observed magnitude of $\Gamma_{I N}$ occurs at $21.0^{\circ} \mathrm{C}$. At higher temperatures, the input impedance deviates from $50 \Omega$ because the temperaturedependent complex permittivity changes the capacitance value of the IDC and thus consequently changes the measured input impedance. Over the measured temperature range, a shift in matched frequency of $0.8 \mathrm{MHz}$ per ${ }^{\circ} \mathrm{C}$ is observed. Apart from a shift in matched frequency, one can observe a flattening of the curve as well. This flattening of the curve complicates the search for the exact matched frequency. The extra uncertainty regarding the exact position of the matched frequency contributes to extra uncertainty regarding the temperature accuracy at higher temperatures.

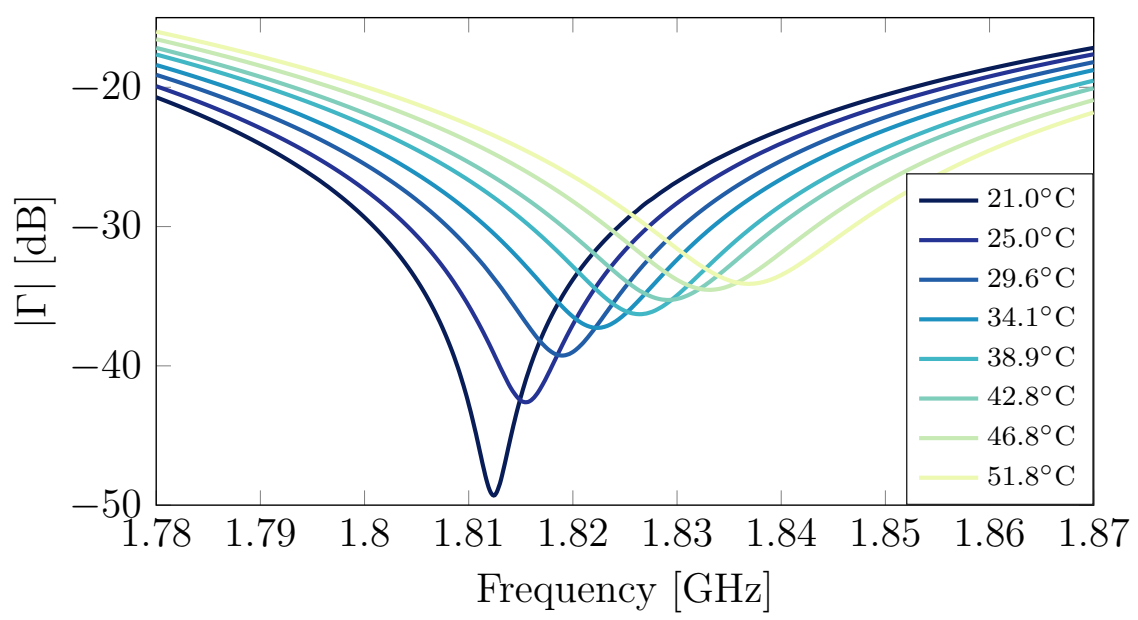

Figure 7: The measured magnitude of the reflection coefficient of the MN terminated with the IDC for multiple temperatures of the water sample. 
Table 2: Polynomial coefficients of the linear fitting $\left(f=p_{0}+p_{1} \times T\right)$ of the obtained data points of the three conducted experiments, together with their corresponding norm of residuals.

\begin{tabular}{|c|c|c|c|}
\hline Experiment & $p_{0}[\mathrm{MHz}]$ & $p_{1}\left[\frac{\mathrm{MHz}}{{ }^{\circ} \mathrm{C}}\right]$ & Norm of residuals \\
\hline 1 & 1795.5 & 0.79631 & 0.59974 \\
\hline 2 & 1795.1 & 0.80683 & 0.30424 \\
\hline 3 & 1795.7 & 0.78844 & 0.44739 \\
\hline
\end{tabular}

After finishing the first of three experiments, the setup was brought again to the initial temperature state, the SMA cable was disconnected, and, the PNA was recalibrated. Afterwards, the same procedure was repeated before the start of the third and final experiment. Throughout all three experiments, a linear response with a slope of $0.8 \mathrm{MHz}$ per ${ }^{\circ} \mathrm{C}$ is observed. The results of each linear fit of all the experiments are shown in Table 2 with their corresponding norm of residuals and they endorse the linear modeling.

At last, we calibrated the system with a linear model to predict the temperatures of the other measurements. Based on two measurements of the first run at a temperature of $21.0^{\circ} \mathrm{C}$ and $51.8^{\circ} \mathrm{C}$, the values $p_{0}$ and $p_{1}$ were determined, respectively $1795.8 \mathrm{MHz}$ and $0.79221 \frac{\mathrm{MHz}}{{ }^{\circ} \mathrm{C}}$, and were used to predict the temperature of all other measurements of experiment 1,2 and 3 based on the measured matched frequency. The resulting temperature calibration curve is shown in Figure 8. Since the absolute accuracy of the calibrated PNA E8361A in reflection tracking in the frequency range of interest is limited to $0.01 \mathrm{~dB}$ (Keysight Technologies 2012), we extracted a frequency range around the minimum of the reflection coefficient $|\Gamma|$ that takes this $0.01 \mathrm{~dB}$ uncertainty effect

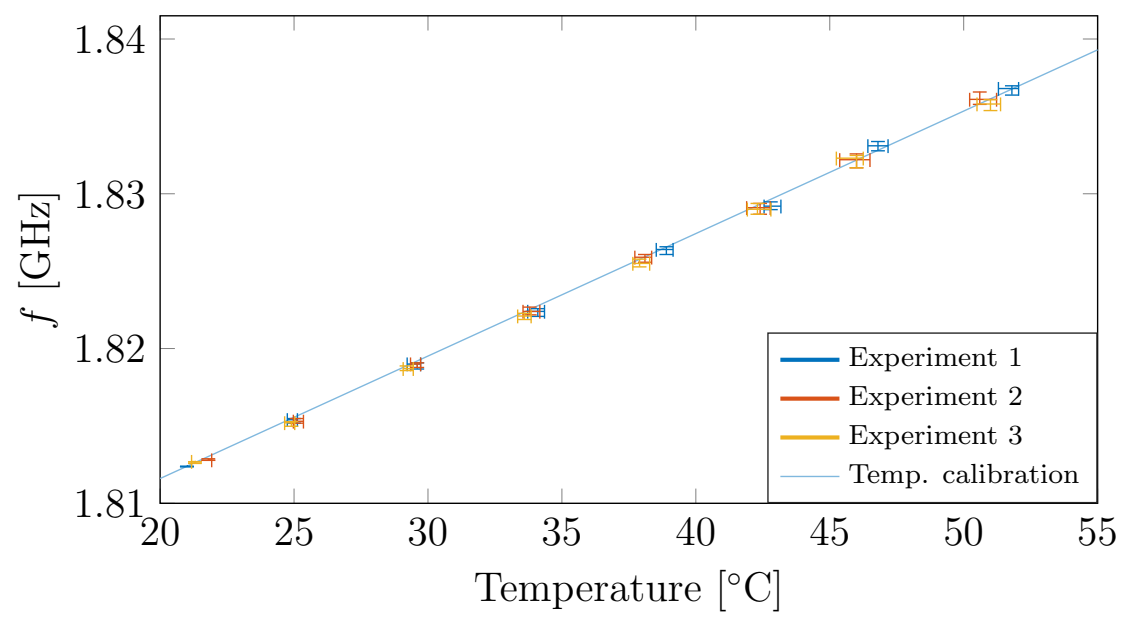

Figure 8: The obtained matched frequencies for the three conducted experiments plotted together with a linear temperature calibration curve based on two data points from the first experiment (i.e. the lowest and the highest temperature point). 


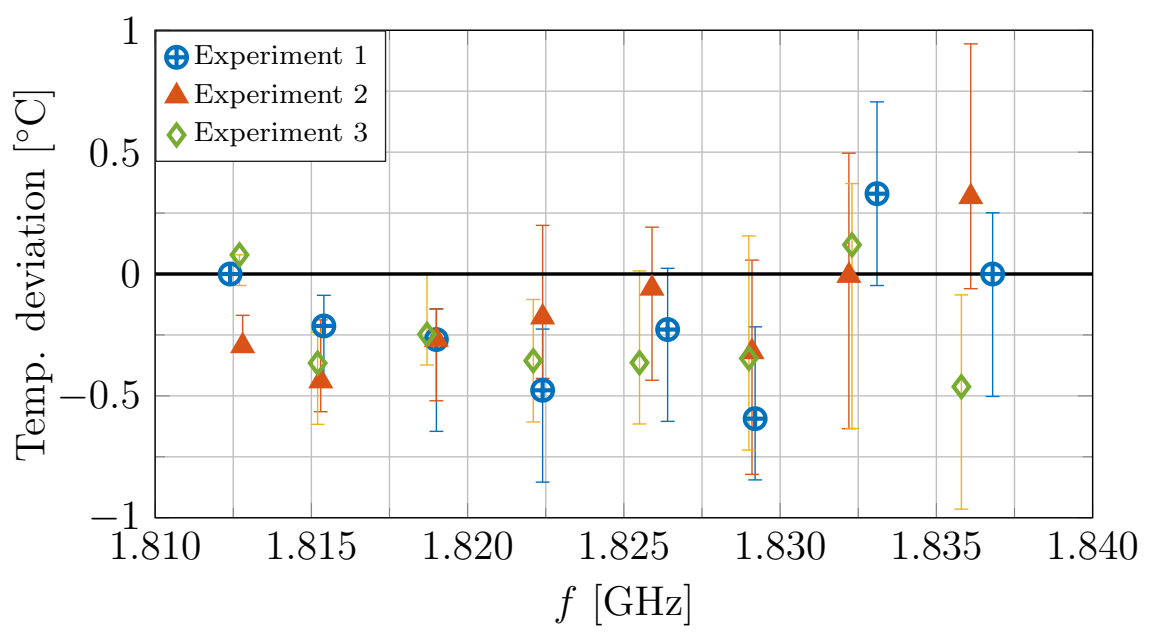

Figure 9: The deviation from the predicted temperature for experiments 1, 2 and 3, with respect to the linear fitted temperature calibration curve based on the lowest and highest temperature points of experiment 1.

into account. Around every measurement point in Figure 8, a vertical uncertainty bar depicts the minimum and maximum frequency of this frequency range and a horizontal uncertainty bar displays the result on the temperature accuracy. The temperature deviation between the predicted and the actual measured temperature is shown in Figure 9. The deviation between the predicted temperature and the actual measured temperature is $\pm 0.24^{\circ} \mathrm{C}$ and has an offset of $-0.19^{\circ} \mathrm{C}$. Once more, the error bars represent the influence of the flattening of the curve on the predicted temperatures.

\section{Discussion and future work}

The presented technique achieved an accuracy of $\pm 0.24^{\circ} \mathrm{C}$ with an offset of $-0.19^{\circ} \mathrm{C}$ in the $20^{\circ} \mathrm{C}$ to $50^{\circ} \mathrm{C}$ temperature range. One can notice as well that the results are more accurate at low temperatures. Since the MN was optimized to be narrow-banded at the lowest temperature, the curve of the magnitude of the reflection coefficient flattens for higher temperatures which results in a less accurate temperature estimation. Nevertheless, the obtained accuracy is adequate and can compete with other existing techniques in microfluidic applications (Table 1). We can conclude that the proposed method presents reliable results and that it is repeatable over multiple conducted experiments. Furthermore, it requires a less advanced control system compared to our previous method since the addition of the $\mathrm{MN}$ results in a linear temperature calibration curve. We did however observe an offset of $-0.19^{\circ} \mathrm{C}$ that could occur from the chosen data points for the linear temperature calibration curve. Due to the limited accuracy of the used type $\mathrm{K}$ reference thermocouple of $\pm 1^{\circ} \mathrm{C}$, we cannot attribute the measured temperature deviations directly to the dielectric-based temperature measurement technique. 
Table 3: Overview of the characteristics of the proposed dielectric-based temperature techniques.

\begin{tabular}{|l||c|c|c|c|c|}
\hline $\begin{array}{l}\text { Measurement } \\
\text { technique }\end{array}$ & $\begin{array}{c}\text { Sensor } \\
\text { placement }\end{array}$ & $\begin{array}{c}\text { Penetration } \\
\text { depth }\end{array}$ & $\begin{array}{c}\text { Temperature } \\
\text { accuracy }\left[{ }^{\circ} \mathrm{C}\right]\end{array}$ & $\begin{array}{c}\text { Thermal time } \\
\text { constant }\end{array}$ & $\begin{array}{c}\text { Calibration } \\
\text { method }\end{array}$ \\
\hline Diel. MN-based & contactless & $60 \mu \mathrm{m}$ & \pm 0.24 & $\mathrm{~N} / \mathrm{A}$ & 2 cal. meas. \\
\hline
\end{tabular}

Table 1 gave an overview of the temperature sensors in literature and the comparison with the novel dielectric-based technique in this work is found in Table 3. It is clear that the presented technique has a similar accuracy as most of the nowadays existing techniques. However, it provides improvements in other domains. First of all, the penetration of the electromagnetic waves in the sample makes this a contactless measurement technique, i.e. it can sense the temperature in the bulk of the sample rather than just at the surface of the sample. Furthermore, this penetration depth can be adjusted during the design of the IDC (the width $W$ and gap $G$ ) (Mamishev et al. 2004). Secondly, since the operating principle of dielectric sensors is directly related to the physical property of the sample under test, there is no need to transfer thermal energy from the sample to the sensor. Therefore, the temperature measurement does not suffer from a thermal time constant, similarly as with infrared radiation. As Tan et al. demonstrated in the case of an RTD, this necessary heat redistribution from the sample to the sensor can take a long time resulting in a long settling time for the RTD up to $100 \mathrm{~s}$ to reach the desired accuracy of $0.1^{\circ} \mathrm{C}$, especially in the case of bad thermal contact (Tan et al. 2008). At last, the technique just requires two calibration measurements due to its linear behavior which is as good as other existing techniques.

It is hard to genuinely compare our results with other temperature-dependent resonators in (Cheng et al. 2012; Lu et al. 2018) due to the large difference in temperature range between $35^{\circ} \mathrm{C}$ in the presented setup and more than $1000^{\circ} \mathrm{C}$ for the known hightemperature sensors. To the authors' best knowledge, only a single paper (Wong et al. 2016) has been presented dealing with a microwave-based temperature sensor for water samples in a microfluidic setup. In this work, we transferred the reusable parts, which enable accurate temperature read-out, off the disposable chip into a permanent setup. This frees up extra space on chip that can be used for cost savings due to space reduction or for adding extra functionality on chip, e.g. microwave biosensing. Additionally, the off-chip components can increase in size. The larger the $\mathrm{MN}$ is, the more narrowbanded it will behave and if the magnitude of the reflection coefficient becomes more narrow-banded, the matched frequency can be obtained with an increased accuracy. The proposed system thus achieves a higher sensitivity of $0.80 \mathrm{MHz}$ per ${ }^{\circ} \mathrm{C}$ compared to $0.33 \mathrm{MHz}$ per ${ }^{\circ} \mathrm{C}$ achieved by Wong et al. (Wong et al. 2016).

In this work, the sample under test was water, one of the most common liquids in biological liquids, buffers and solutions. Thus, as expected, water-based solutions also demonstrate a similar temperature-dependent dielectric behavior, e.g. sodium chloride solutions at different concentrations (Peyman et al. 2007). Additionally, the permittivity 
of other liquids, e.g. 2-propanol, is also temperature-dependent as demonstrated by Sato et al. (Sato and Buchner 2003) in the temperature range from $20^{\circ} \mathrm{C}$ to $30^{\circ} \mathrm{C}$. Therefore, the operating principle behind this technique is also valid for temperature sensing of different liquids provided that the calibration step is changed accordingly.

\section{Conclusion}

In this work, we presented a dielectric-based temperature sensing technique. The presented technique evolved from our previous work and introduces a matching network to the system. This matching network matches the capacitive sensor, with a sample at a specific temperature, at a specific frequency. This technique relaxes the computational power required with our previous method and it can be calibrated with a linear curve requiring only two calibration measurement. The technique proved to be stable throughout three experiments and it achieved an accuracy of $\pm 0.24^{\circ} \mathrm{C}$ with an offset of $-0.19^{\circ} \mathrm{C}$ in a $20^{\circ} \mathrm{C}$ to $50^{\circ} \mathrm{C}$ temperature range. In addition, no physical contact is required and the temperature of the sample is measured volumetrically in a labelfree manner. The combination of dielectric-based temperature sensing with well-known microwave heating can lead to dual-purpose devices for temperature control units while achieving a very efficient footprint on a microfluidic chip.

\section{Acknowledgments}

This work is sponsored by the KU Leuven $\mathrm{C} 2\left(\mu^{2} \mathrm{BIO}\right)$ project funding and is supported in part by the Research Foundation Flanders (FWO) SB PhD fellowship under grant number 1S23918N (Gertjan Maenhout). The authors would like to thank their colleagues at KU Leuven Div. Telemic for productive discussions as well as constructive feedback and FabLab Leuven for access to their laser cutters for the fabrication of the PMMA and wood panels.

\section{References}

J. Bao, T. Markovic, I. Ocket, D. Kil, L. Brancato, R. Puers, and B. Nauwelaers. Investigation of thermal effect caused by different input power of biosensor using a novel microwave and optical sensing system for biological liquids. In Proceedings of the 1st IEEE MTT-S IMBioC, pages 1-4, May 2017.

J. Bao, S. Yan, T. Markovic, I. Ocket, D. Kil, L. Brancato, R. Puers, and B. Nauwelaers. A 20-ghz microwave miniaturized ring resonator for $\mathrm{nl}$ microfluidic sensing applications. IEEE Sensors Letters, 3(6):1-4, June 2019.

J. Bao, G. Maenhout, T. Markovic, I. Ocket, and B. Nauwelaers. A microwave platform for reliable and instant interconnecting combined with microwave-microfluidic interdigital capacitor chips for sensing applications. Sensors, 20(6):1687, 2020. 
A. S. Basu and Y. B. Gianchandani. Shaping high-speed marangoni flow in liquid films by microscale perturbations in surface temperature. Applied Physics Letters, 90(3): $034102,2007$.

K. Bhargava, B. Thompson, A. Tembhekar, and N. Malmstadt. Temperature sensing in modular microfluidic architectures. Micromachines, 7(1):11, 2016.

H. Cheng, S. Ebadi, and X. Gong. A low-profile wireless passive temperature sensor using resonator/antenna integration up to $1000^{\circ} \mathrm{C}$. IEEE Antennas and Wireless Propagation Letters, 11:369-372, 2012. ISSN 1536-1225.

W. J. Ellison. Permittivity of pure water, at standard atmospheric pressure, over the frequency range $0-25 \mathrm{THz}$ and the temperature range $0-100^{\circ} \mathrm{C}$. Journal of Physical and Chemical Reference Data, 36(1), 2007.

R. Esfandyarpour, M. J. Didonato, Y. Yang, N. G. Durmus, J. S. Harris, and R. W. Davis. Multifunctional, inexpensive, and reusable nanoparticle-printed biochip for cell manipulation and diagnosis. Proceedings of the National Academy of Sciences, 114(8):1306-1315, Jun 2017.

L. Estel, M. Poux, N. Benamara, and I. Polaert. Continuous flow-microwave reactor: Where are we? Chemical Engineering and Processing - Process Intensification, 113: 56-64, 2017.

J. Feng, M. Ding, J. Kou, F. Xu, and Y. Lu. An optical fiber tip micrograting thermometer. IEEE Photonics Journal, 3(5):810-814, Oct 2011. ISSN 1943-0655.

F. Gillot, A. Tixier-Mita, F. Morin, and H. Fujita. High response time micro scale thermo-couple for biological applications. In 2006 International Conference on Microtechnologies in Medicine and Biology, pages 127-130, May 2006.

K. Grenier, D. Dubuc, P. Poleni, M. Kumemura, H. Toshiyoshi, T. Fujii, and H. Fujita. Integrated broadband microwave and microfluidic sensor dedicated to bioengineering. IEEE Transactions on Microwave Theory and Techniques, 57(12):3246-3253, Dec 2009. ISSN 0018-9480.

T. Kaji, T. Kawashima, M. Sakamoto, Y. Kurashige, and F. Koizumi. Inhibitory effect of Rhodamine B on the proliferation of human lip fibroblasts in culture. Toxicology, 68(1):11-20, 1991.

K. Keränen, J.-T. Mäkinen, P. Korhonen, E. Juntunen, V. Heikkinen, and J. Mäkelä. Infrared temperature sensor system for mobile devices. Sensors and Actuators A: Physical, 158(1):161-167, 2010.

Keysight Technologies. Keysight E8361A/C PNA Network Analyzer. 122012.

H. Li, L. Ma, Q. Zhao, F. Zhang, C. Wang, and X. Liu. Rapid response of high precision fiber bragg grating based temperature sensor. In 2018 IEEE 3rd Optoelectronics Global Conference (OGC), pages 64-67, Sep. 2018.

F. Lu, Q. Tan, Y. Ji, Q. Guo, Y. Guo, and J. Xiong. A novel metamaterial inspired high-temperature microwave sensor in harsh environments. Sensors, 18(9), 2018. ISSN 1424-8220. 
G. Maenhout, J. Bao, T. Markovic, I. Ocket, and B. Nauwelaers. Reliable, fast and reusable interfacing of high-frequency signals to disposable lab-on-a-chip devices. In Proceedings of the 3rd IEEE MTT-S IMBioC, volume 1, pages 1-4, May 2019a.

G. Maenhout, T. Markovic, J. Bao, G. Stefanidis, I. Ocket, and B. Nauwelaers. Reliable, fast and reusable interfacing of high-frequency signals to disposable lab-on-a-chip devices. In The 4th Conference on Microfluidic Handling Systems, pages 112-115, October 2019b.

A. V. Mamishev, K. Sundara-Rajan, F. Yang, Y. Du, and M. Zahn. Interdigital sensors and transducers. Proceedings of the IEEE, 92(5):808-845, May 2004. ISSN 0018-9219.

T. Mandic, R. Gillon, B. Nauwelaers, and A. Baric. Characterizing the TEM Cell Electric and Magnetic Field Coupling to PCB Transmission Lines. IEEE Transactions on Electromagnetic Compatibility, 54(5):976-985, Oct 2012. ISSN 0018-9375.

D. Mark, S. Haeberle, G. Roth, F. von Stetten, and R. Zengerle. Microfluidic lab-ona-chip platforms: requirements, characteristics and applications. Chemical Society reviews, 39:1153-1182, 2010.

T. Markovic, I. Ocket, B. Jones, and B. Nauwelaers. Characterization of a novel microwave heater for continuous flow microfluidics fabricated on high-resistivity silicon. In 2016 IEEE MTT-S International Microwave Symposium (IMS), pages $1-4,2016$.

V. Miralles, A. Huerre, F. Malloggi, and M.-C. Jullien. A review of heating and temperature control in microfluidic systems: Techniques and applications. Diagnostics, 3(1):33-67, 2013. ISSN 2075-4418.

M. I. Mohammed, S. Haswell, and I. Gibson. Lab-on-a-chip or chip-in-a-lab: Challenges of commercialization lost in translation. Procedia Technology, 20:54-59, 2015.

A. Peyman, C. Gabriel, and E. Grant. Complex permittivity of sodium chloride solutions at microwave frequencies. Bioelectromagnetics, 28(4):264-274, 2007.

D. M. Pozar. Microwave engineering. John Wiley \& Sons, $4^{\text {th }}$ edition, 2012.

J. A. Reynoso-Hernández, C. F. Estrada-Maldonado, T. Parra, K. Grenier, and J. Graffeuil. An improved method for the wave propagation constant $\gamma$ estimation in broadband uniform millimeter-wave transmission line. Microwave and Optical Technology Letters, 22(4):268-271, 1999. ISSN 1098-2760.

D. Ross, M. Gaitan, and L. E. Locascio. Temperature measurement in microfluidic systems using a temperature-dependent fluorescent dye. Analytical chemistry, 73(17): 4117-4123, 2001.

M. Ryu, J. Kimber, T. Sato, R. Nakatani, T. Hayakawa, M. Romano, C. Pradere, A. Hovhannisyan, S. Kazarian, J. Morikawa, and et al. Infrared thermo-spectroscopic imaging of styrene radical polymerization in microfluidics. Chemical Engineering Journal, 324:259-265, 2017.

T. Sato and R. Buchner. Dielectric relaxation spectroscopy of 2-propanolwater mixtures. Journal of Chemical Physics, 118(10):4606-4613, 2003. 
M. Shibata, T. Yamaguchi, S. Kumagai, and M. Sasaki. Thermocouples on trench sidewall in channel fronting on flowing material. In Proceedings of the 28th IEEE International Conference on Micro Electro Mechanical Systems, pages 841-844, Jan 2015.

W. W. Tan, R. F. Y. Li, A. P. Loh, and W. K. Ho. Rtd response time estimation in the presence of temperature variations and its application to semiconductor manufacturing. IEEE Transactions on Instrumentation and Measurement, 57(2):406412, Feb 2008.

D. Wong, G. Yesiloz, M. S. Boybay, and C. L. Ren. Microwave temperature measurement in microfluidic devices. Lab on a Chip, 16(12):2192-2197, 2016.

J. Wu. A basic guide to thermocouple measurements. In Texas Instruments, page 137, 2018.

Y.-F. Yap, S.-H. Tan, N.-T. Nguyen, S. M. S. Murshed, T.-N. Wong, and L. Yobas. Thermally mediated control of liquid microdroplets at a bifurcation. Journal of Physics D: Applied Physics, 42(6):065503, Feb 2009.

G. Yesiloz, M. S. Boybay, and C. L. Ren. Effective thermo-capillary mixing in droplet microfluidics integrated with a microwave heater. Analytical Chemistry, 89(3):19781984, 2017.

W. J. Yoo, D. H. Cho, K. W. Jang, S. H. Shin, J. K. Seo, S.-C. Chung, G.-R. Tack, B. Lee, B. G. Park, J. H. Moon, and S. Kim. Infrared fiber-optic sensor for non-contact temperature measurements. In Proceedings of the 3rd International Conference on Sensing Technology, pages 500-503, Nov 2008.

R. Zhong, X. Pan, L. Jiang, Z. Dai, J. Qin, and B. Lin. Simply and reliably integrating micro heaters/sensors in a monolithic PCR-CE microfluidic genetic analysis system. Electrophoresis, 30(8):1297-1305, 2009. 